\title{
RYODORAKU TREATMENT OF ENURESIS NOCTURNA
}

\author{
Masuto Misawa \\ Director of the Japan Ryodoraku Autonomic Nerve Society and \\ Vice-Director of Shironishi Hospital
}

\section{Introduction}

The term enuresis nocturna, as defined by Moriya, means urine incontinence or bedwetting which occurs more than 2 to 3 times at night every day or several days in the week after age 3 .

When enuresis nocturna occurs due to organic disorders, such as urethral stone, malformation, inflammation, spinal bifida and disturbances of the central nervous system and hormone secretion, a relatively high rate of curing can be achieved by treating the causative disease. However, as the vast majority (90-93\%) of cases of enuresis nocturna is due to functional defect or disease and is very hard to cure.

The authors have employed Ryodoraku therapy in the treatment of enuresis nocturna due to functional defect and obtained favorable results.

\section{Sumary of Needle Treatment}

Enuresis nocturna has been treated by needle therapy in China, and in Japan Nakatani has been treating it by electric needle therapy. Both therapies consist in the insertion of needles into points. The points have close relation with the nerve which governs the bladder so that when electric needles are inserted into the intersections of the points and the nerve, a better therapeutic result can be obtained.

\begin{abstract}
Anatomy
The bladder will be briefly described from an anatomical point of view, for some anatomical knowledge of the bladder is necessary to practice this treatment. According to Okajima, Taniguchi et al., the bladder wall consists of three layers. Its activity is governed by the spinal and autonomic nerves. The periphery of the spinal nerve governs the lateral and frontal portions of the bladder, and the autonomic nerve also reaches the bladder, urethra and ureter. The spinal and autonomic nerves are connected with each other by communications branches.
\end{abstract}

\section{Apparatus}

The apparatus used in this treatment is a Neurometer and an electric current of 12 volts, $150-200 \mu \mathrm{A}$ is applied for 10 seconds intermittently in most cases.

\section{Technique}

A needle is inserted horizontally into the point about $2.0-2.5 \mathrm{~cm}(4.0-4.5 \mathrm{~cm}$ in adults) laterally apart from the lumbar spine to the depth of about $2.0-2.5 \mathrm{~cm}(4.0-$ 
$4.5 \mathrm{~cm}$ in adults), aiming at the lumbar median line, with electric stimulation. If the target is hit, the patient has a sensation of numbness at the site. In the same manner, needles are anserted into the nerves of No. 1-No. 5 lumbar vertebrae and sacral foramen. (this 2nd foramen is the most important point, so main nerve of bladder is governed by 2 nd sacral nerve).

In the abdomen, a better effect can be obtained, if some needles are inserted directly into the bladder, but utmost care should be used in the sterilization of the needles.

\section{Psychotherapy}

In the treatment of enuresis, psychotherapy should always be practiced in parallel with the treatment mentioned above. It is important to find and remove the psychologic factors which are held responsible for enuresis. Besides, exercise and other treatments are also combined.

\section{Therapeutic Effects}

Of tht 100 subjects treated, 3 cases were at age $4,6 \mathrm{c}$. at age $5,21-6,5-8,13-10$, $18-11,28-12,3-15,1-18,1-21$, and 1 case at age 28 .

As for the frequency of urine incontinence, $70 \%$ of the subjects wetted the beds every night and $30 \%$ of the subjects did 2 to 3 times every week. Treatment was given 2 to 3 times a week, and it was not repeated more than 5 times in all. The therapeutic effects were evaluated on the basis of the findings obtained by a follow-up and chart records.

As the result of treatment, $71 \%$ of the subjects were completely cured, $15 \%$ of the subjects were improved, and $14 \%$ of the subjects remained unchanged.

During the treatment all the patients were given intramuscularly 1 vial of vitamin $B_{12}$ (Methicobal) and $2-3$ vials of viatmin $\mathrm{B}_{6}(\mathrm{BZ})$

\section{Discussion}

Enuresis nocturna could be either cured or improved by Ryodoraku therapy in a relatively short time. Psychotherapy and injection therapy which were combined with Ryodoraku therapy may account for some part of the effect, but most of this effect can be explained by the direct or indirect stimulation of the spinal and autonomic nerves which have a direct relation with the bladder wall. This stimulation increases the tonus of the bladder wall muscle and sphincter so that the patient restors a normal sensation of the full bladder. Thanks for Prof. Dr. Hiroshi Tsukagoshi of Shinshu-University.

\section{References}

1) Togawa, Y. et al. : Abnormal characters and therapy, Kaneko Shobo, 4, 21, 1973

2) Chinese Medicine : Shanghai People's Publishing Corp., 489, 1972

3) Okajima, K. and Taniguchi, T. : Anat., Tofudo, 270-271, 1914

4) Misawa, M. : An easy guide to the spots of pain, Tokyo Ryodoraku Autonomic Nervous System Research Institute, 20, 1974 